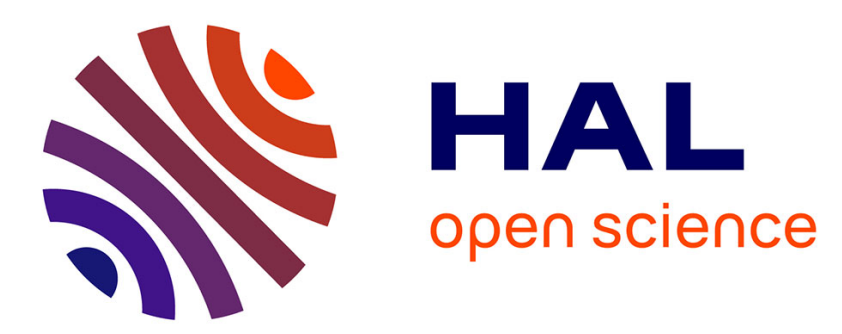

\title{
Axial hypertoroidal moment in a ferroelectric nanotorus: A way to switch local polarization
}

\author{
G. Thorner, Jean-Michel Kiat, Christine Bogicevic, I. Kornev
}

\section{To cite this version:}

G. Thorner, Jean-Michel Kiat, Christine Bogicevic, I. Kornev. Axial hypertoroidal moment in a ferroelectric nanotorus: A way to switch local polarization. Physical Review B: Condensed Matter and Materials Physics (1998-2015), 2014, 89, pp.220103. 10.1103/physRevB.89.220103 . hal-01053344

\section{HAL Id: hal-01053344 https://hal.science/hal-01053344}

Submitted on $30 \mathrm{Jul} 2014$

HAL is a multi-disciplinary open access archive for the deposit and dissemination of scientific research documents, whether they are published or not. The documents may come from teaching and research institutions in France or abroad, or from public or private research centers.
L'archive ouverte pluridisciplinaire HAL, est destinée au dépôt et à la diffusion de documents scientifiques de niveau recherche, publiés ou non, émanant des établissements d'enseignement et de recherche français ou étrangers, des laboratoires publics ou privés. 


\title{
Axial hypertoroidal moment in a ferroelectric nanotorus: A way to switch local polarization
}

\author{
Gentien Thorner, ${ }^{1}$ Jean-Michel Kiat,,${ }^{1,2}$ Christine Bogicevic, ${ }^{1}$ and Igor Kornev ${ }^{1}$ \\ ${ }^{1}$ Laboratoire Structures, Propriétés et Modélisation des Solides, associé au CNRS (UMR8580), Ecole Centrale Paris, \\ Grande Voie des Vignes, 92295 Châtenay-Malabry, France \\ ${ }^{2}$ Laboratoire Léon Brillouin, associé au CNRS (UMR12), CEA Saclay, 91191 Gif-sur-Yvette, France
}

(Received 24 December 2013; revised manuscript received 16 April 2014; published 6 June 2014)

\begin{abstract}
Growth of ferroelectric nanotori is reported and first-principles-based effective Hamiltonian simulations were performed on these new objects. They could reproduce the nonpolar (phase I) and homogeneously toroidized (phase II) states of an isolated nanotorus. Computation of an axial hypertoroidal moment leads to numerical observation of two new phases: (i) a homogeneously hypertoroidized one (phase III) that can be switched by a homogeneous electric field and (ii) another one with striking new polarization patterns (phase IV) due to azimuthal variations of the hypertoroidal moment. In both phases, hypertoroidization coexists with a homogeneous axial toroidal moment.
\end{abstract}

DOI: 10.1103/PhysRevB.89.220103

PACS number(s): 64.60.-i, 61.46.-w, 77.80.B-

A giant step in the field of ferroelectricity occurred with the discovery of toroidal ordering [1] of polarization [2]. Indeed the possibility to create new dipole configurations based on this special arrangement of polar order in the form of vortices has opened exciting possibilities for the downscaling of electronic devices such as ferroelectric random access memories [3]. However, as ferroelectric toroidization is an axial vector, it is unaffected by homogeneous electric fields: Contrary to overall polarization, there is no possibility of switching by a classical way. Thus, writing memory nanodevices based on toroidal moments would require complex solutions.

One possible way could be to use nanodots having the shape of nanorings with an off-centered hole. In these objects, the transverse hypertoroidal moment (which is a polar vector [4]) has been extensively studied by Prosandeev et al. [5] at room temperature and above. This order parameter involves a double cross product of local polarization with dipole site positions, in order to probe the curl of ferroelectric toroidization. This provides a convenient way of describing states that have subtle ordering features which cannot be described in terms of overall polarization or toroidization. As an example, computation of this parameter can probe the curvature in flower states or characterize onion states. From a more general point of view, this parameter shares the time-reversal symmetry and space-reversal antisymmetry properties of homogeneous polarization and, being a polar vector, can couple to homogeneous electric fields.

Perovskite nanorings with an off-centered hole as in [5] are most probably very difficult to obtain and, to the best of our knowledge, their synthesis has not been reported. Therefore, in our study, our purpose is to keep the torus hole at the center for technological reasons, and to demonstrate that another component of the hypertoroidal moment, related to a completely different inhomogeneous polarization state, can be switched, i.e., we focus on the axial part of the hypertoroidal moment that describes poloidal polarization in a torus.

Thus, this Rapid Communication will first explain the general interest of ferroelectric nanotori. Then, it will move on to the $\mathrm{BaTiO}_{3}$ nanotori that we experimentally obtained. In the rest of this Rapid Communication, deeper insight will be gained with effective Hamiltonian simulations of local polarization: Axial hypertoroidal moment will be introduced and the effect of the torus shape will be investigated. Spontaneous azimuthal modulation of an axial hypertoroidal moment will be evidenced. Lastly, in the case where the axial hypertoroidal moment is homogeneous, switching by homogeneous electric fields will be presented.

Beyond simply connected geometries such as zerodimensional dots [2] and hysterons [6] (elongated nanodots) or one-dimensional wires $[7,8]$ or two-dimensional planes $[9,10]$, torus-shaped nanodots (or nanorings) give rise to new and fascinating dipolar patterns. Another advantage of nanotori is the fact that their geometry makes them unlikely to form homogeneously polarized states in which free charges screen the polarization. Moreover, nanoring geometry prevents isolated dots with finite toroidal moments to get strain energy penalty due to the presence of a polarization topological defect at the vortex core [11], as this defect is in this case outside of the ferroelectric material. Last but not least, bowl-like $\mathrm{BaTiO}_{3}$ nanoparticles have been experimentally obtained [12] without using any template [13].

In this work, hydrothermal growth of $\mathrm{BaTiO}_{3}$ nanotori is reported, starting from hydrothermally prepared titanate multiwall spiral nanotubes [14]. Contrary to the case considered in [15], the crystal structure induces anisotropy and the actual geometry is shown in Fig. 1.

Based on the transmission electron microscopy (TEM) observation of the edges and on that of larger regions, the rest of this work deals with simulations describing a single-crystalline stress-free torus whose main axis lies along the pseudocubic [001] direction, subsequently called the $z$ axis, with unit vector $\mathbf{e}_{z}$.

All the numerical results were obtained with the coefficients of the first-principles-derived effective Hamiltonian [16] of bulk $\mathrm{BaTiO}_{3}$ : For the whole simulation cell (1), it depends on all the polar local modes $\left(\mathbf{u}_{i}=\mathbf{p}_{i} / Z^{*}\right.$ with Born effective charge $\left.Z^{*}\right)$ and on all the local strains of each $i$ th five-atom unit cells.

$$
\begin{aligned}
E^{\text {tot }}= & E^{\text {self }}(\{\mathbf{u}\})+E^{\text {dpl }}(\{\mathbf{u}\})+E^{\text {short }}(\{\mathbf{u}\}) \\
& +E^{\text {elas }}\left(\left\{\eta_{l}\right\}\right)+E^{\text {int }}\left(\{\mathbf{u}\},\left\{\eta_{l}\right\}\right) .
\end{aligned}
$$



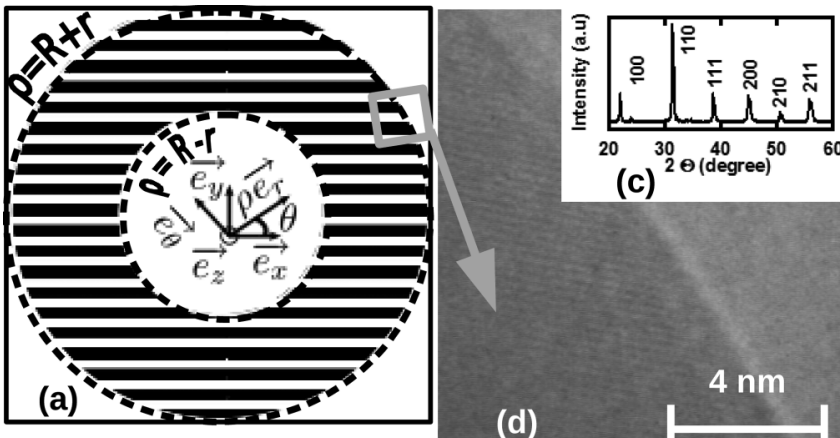

(c)
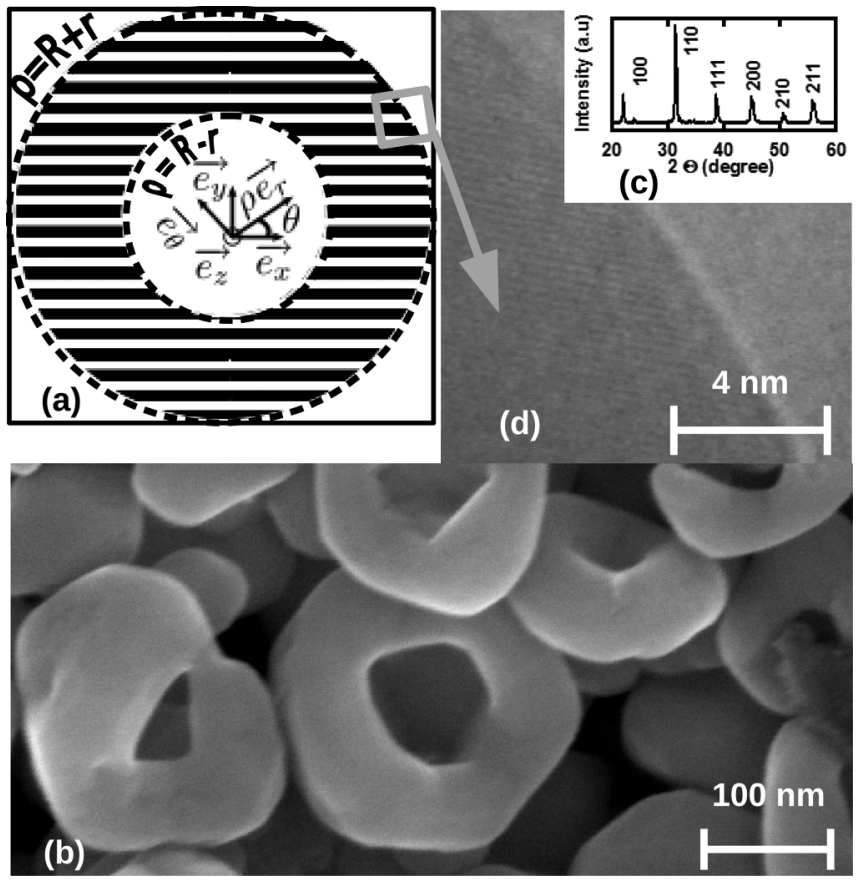

FIG. 1. (a) Highly anisotropic experimentally obtained and simulated torus with major radius $R$ and minor radius $r$. (b) $\mathrm{BaTiO}_{3}$ nanotori scanning electron microscopy picture. (c) X-ray diffractogram. (d) Transmission electron microscopy image of lattice fringes at the edge of a nanotorus.

The first term in Eq. (2) gives rise to an energy penalty when polarization does not point along the principal axes and, as such, takes the anisotropy of the structure drawn in Fig. 1 into account.

$$
\begin{aligned}
E^{\text {self }}(\{\mathbf{u}\})= & \sum_{i}\left[\kappa_{2}\left|\mathbf{u}_{i}\right|^{2}+\alpha\left|\mathbf{u}_{i}\right|^{4}\right. \\
& \left.+\gamma\left(u_{i x}^{2} u_{i y}^{2}+u_{i y}^{2} u_{i z}^{2}+u_{i z}^{2} u_{i x}^{2}\right)\right] .
\end{aligned}
$$

Monte Carlo (MC) simulations were performed using a Metropolis-Hastings algorithm. At each temperature, 50000 MC sweeps were performed, and physical observables were recorded over 50000 extra averaging sweeps. Cooling steps were $50 \mathrm{~K}$ from 950 to $200 \mathrm{~K}$ and $5 \mathrm{~K}$ afterwards.

The high anisotropy of nanotorus imposed a parallelepipedic simulation cell [represented with a solid line in Fig. 1(a)] in which dipole sites form a cubic grid. However, inside these supercells, the lattice sites that sit outside of the torus do not contain ferroelectric material so their local dipoles were permanently set to zero and their elastic constants matched to those of the ferroelectric. To account for the fully unscreened electrostatic boundary conditions around the isolated particle, dipole-dipole interaction was computed by real-space summation over the simulation cell rather than the Ewald method [17]. No surface-induced relaxation term was included. The simulation supercell had periodic elastic boundary conditions, so that the local strain was not imposed at the edges. During this study, the number of ferroelectric lattice sites inside the simulation supercell were kept approximately constant at a value of $N=2400$. However, as the aspect ratio $a=r / R$ of the torus enclosed in the supercell is a control parameter as we show below, various simulation boxes, shapes, and sizes were used in the present work (exact sizes were $23 \times 23 \times 12,27 \times 27 \times 11,28 \times 28 \times 10$, $38 \times 38 \times 9,42 \times 42 \times 8$, and $77 \times 77 \times 7$, in units of lattice constant, which means $v^{1 / 3} \approx 4 \AA$ ).

Several global quantities were recorded, such as homogeneous volumic polarization [expression of $\mathbf{P}$ in Eq. (3), in which index $i$ runs along all lattice sites contained in the supercell], which allows computation of transverse polarization at site $i, \delta \mathbf{p}_{i}=\mathbf{p}_{i}-v \mathbf{P}$.

$$
\begin{aligned}
\mathbf{P} & =\frac{1}{N v} \sum_{i} \mathbf{p}_{i}, \\
\mathbf{T} & =\frac{1}{2 N v} \sum_{i} \mathbf{r}_{i} \times \delta \mathbf{p}_{i}, \\
\mathbf{H}_{e} & =\frac{1}{4 N v} \sum_{i}\left[\mathbf{r}_{i} \times\left(\mathbf{r}_{i} \times \delta \mathbf{p}_{i}\right)\right] .
\end{aligned}
$$

Even though both of the global quantities mentioned thereafter have exact expressions that are origin independent [18], we used a supercell-centered set of coordinates to write the electric toroidal moment $\mathbf{T}$ (4) and a simplified form [19] of the electric hypertoroidal moment $\mathbf{H}_{e}$ (5).

Toroidal moment physically describes the average of the curl of the polarization vector field, whereas the less intuitive the hypertoroidal moment represents the average of the curl of the toroidization vector field [4]. Thus, in the geometry of a torus, the axial component of overall toroidal moment $T_{z}=$ $\mathbf{T} \cdot \mathbf{e}_{z}$ directly probes the toroidal component of polarization field lines (i.e., local ordering of dipoles along $\mathbf{e}_{\theta}$ ), whereas overall axial hypertoroidal moment $H_{e z}=\mathbf{H}_{e} \cdot \mathbf{e}_{z}$ directly probes the poloidal component of polarization field lines (i.e., local ordering of toroidal moments along $\mathbf{e}_{\theta}$ ) [20]. Therefore, this $z$-axis component of the hypertoroidal moment in nanotori is completely different from its $x y$-plane components in rings $[4,5,18]$ having an off-centered hole.

In this study, we have investigated the effect of torus aspect ratio $a$ which is the main control parameter. Figure 2(a) shows that four phases arise when cooling an isolated nanotorus of arbitrary aspect ratio. In the absence of externally applied homogeneous electric fields, all components of homogeneous polarization and transverse toroidization $\left(T_{x}\right.$ and $\left.T_{y}\right)$ remained zero within error bars at the temperatures and aspect ratios we plotted.

At high temperatures, a paraelectric phase with no toroidal moment (phase I with field line pattern symmetry point group $\infty / \mathrm{mmm}$, even though the dipole grid has $4 / \mathrm{mmm}$ symmetry) is always observed whatever the $a$ value is [Fig. 2(b)]. It always converts at lower temperatures into a ferrotoroidic state (phase II, field line pattern symmetry point group $\infty / m$ ). These two phases were already known since they have also been observed by Prosandeev et al. in lead zirconate titanate nanorings $[4,5,18]$.

Our simulations reveal two additional phases: At low temperatures, the polarization state depends on the $a$ value [Figs. 2(f)-2(h)]. In the $a>0.3$ case, phase II converts 

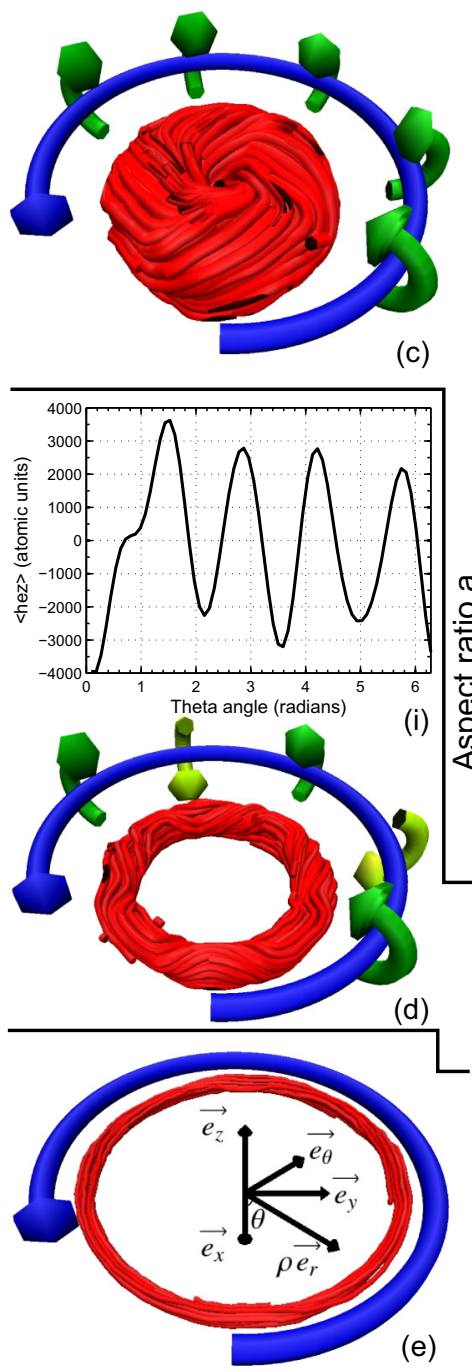

e)
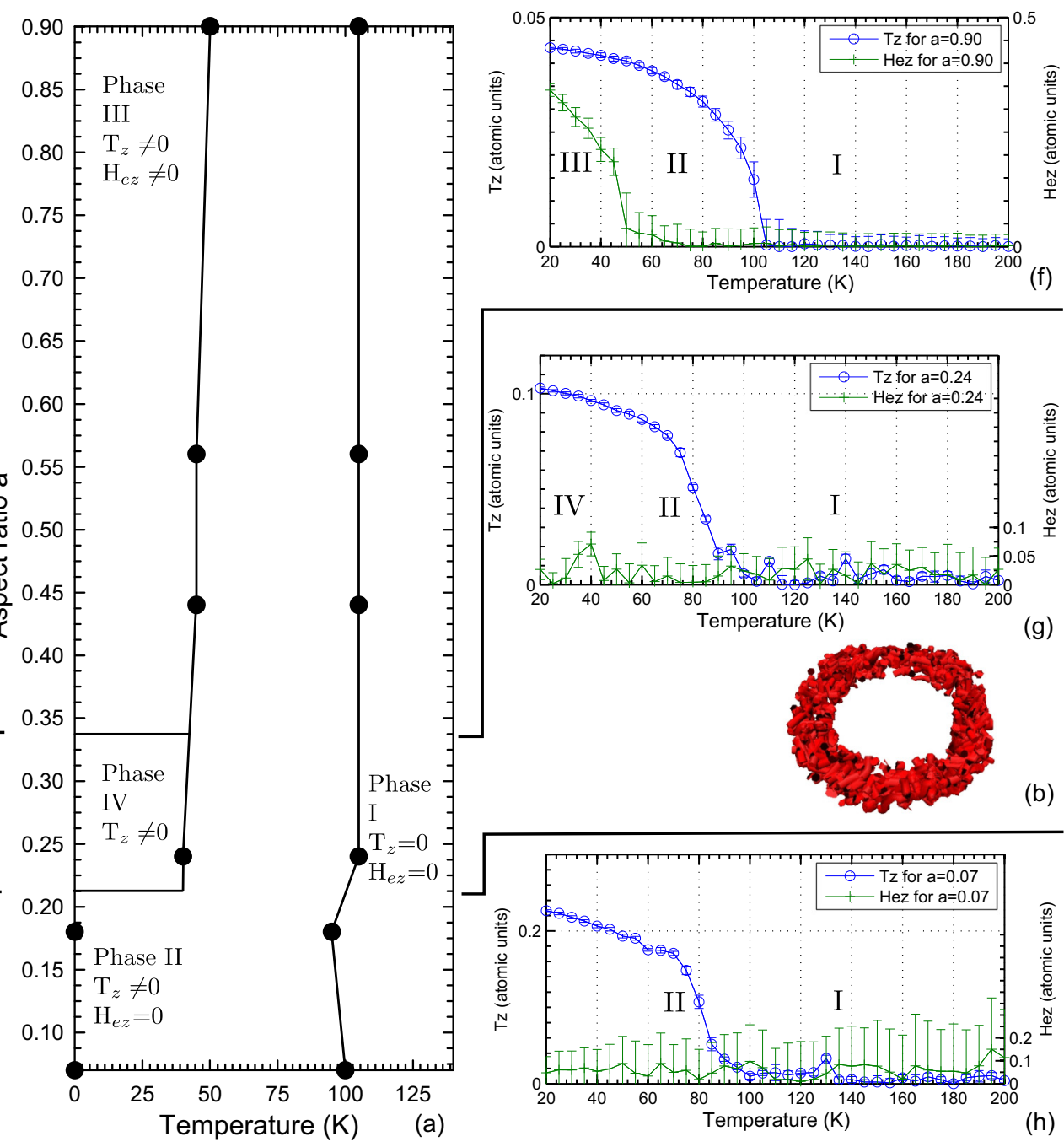

(g)

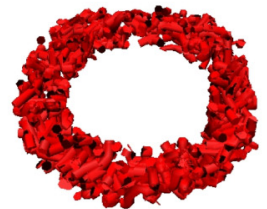

(b)

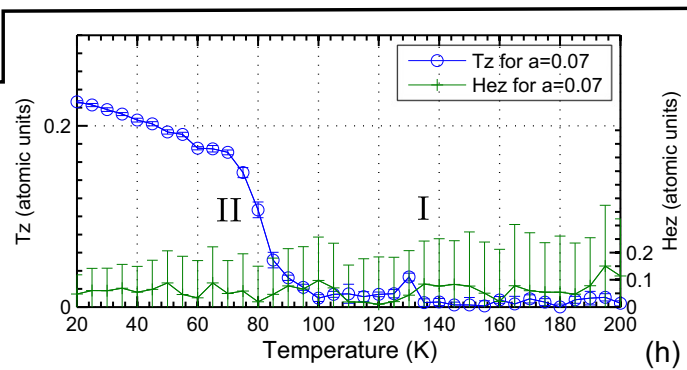

(h)

FIG. 2. (Color online) (a) Aspect ratio versus temperature phase diagram. (b) Red polarization field lines of high temperature nonpolar phase I at $450 \mathrm{~K}$ for $a=0,24$. (c)-(e) Red polarization field lines at $5 \mathrm{~K}$ for $a=0.90$ (phase III), 0.24 (phase IV), and 0.07 (phase II); horizontal blue arrow represents toroidal moment; vertical green arrows represent hypertoroidal moment. (f)-(h) plot axial toroidal (blue circles) and hypertoroidal (green crosses) moments as functions of temperature for the same values of $a$. In phase IV, (i) represents the oscillations of hypertoroidization as a function of azimut $\theta$ at $5 \mathrm{~K}$ for $a=0.24$.

into another state that has both toroidal and hypertoroidal moments along the $z$ direction (phase III, field line pattern point group $\infty$ ) [Fig. 2(c)]. Even though the dipole grid has $4 / \mathrm{mmm}$ symmetry, considering that rotating a hysteron around its axis generates a solid spindle torus, there is an analogy between the poloidal component of polarization and the double vortex states that have been simulated in isolated hysterons [6]. However, polarization field lines are not purely poloidal, as expected in [21-23]. There is a coexistence of axial toroidal moment with axial hypertoroidal moment that reminds one of the coexistence of homogeneous polarization with toroidal moment that is also observed along the main axis of an array of aligned infinite ferroelectric nanowires $[8,24]$. On the contrary, at low values of $a<0.2$, there is no second transition, and phase II remains stable down to low temperatures [Fig. 2(e)]. For an intermediate value $0.2<a<0.3$, the competition between these two interactions produces interesting new phenomena. Indeed, in that case, neither phase II nor phase III can be stable, and this frustrated situation leads to unexpected symmetries in the polarization patterns [Fig. 2(d)]. On average, the new phase IV has no hypertoroidal moment at low temperatures, as was the case for phase II. However, its polarization field lines at low temperature do not exhibit the same behavior as in phase II: The absolute value of local hypertoroidal moment along $z\left(h_{e z i}=1 / 4\left[\mathbf{r}_{i} \times\left(\mathbf{r}_{i} \times \mathbf{p}_{i}\right)\right] \cdot \mathbf{e}_{z}\right)$ is finite in most of the torus. Cancellation of average $H_{e z}$ only occurs due to azimuthal oscillations of $h_{e z i}$. Drawing an analogy with the situation observed in antiferroelectrics/antiferromagnetics, phase IV can be described as antiferrohypertoroidic, in addition to its ferrotoroidic component. Deeper insight in phase IV requires an understanding of these variations. Thus, at $5 \mathrm{~K}$ for $a=0.24$, we plotted in Fig. $2(\mathrm{~g})$ a truncated series $(K=7)$ that captured the oscillations [Fig. 2(d)] of 
hypertoroidization (6).

$$
\begin{aligned}
& \left\langle h_{e z}\right\rangle_{\rho, z}(\theta) \\
& =\frac{1}{2 K+1} \sum_{i} h_{e z i}\left[1+2 \sum_{k=1}^{K}\left(T_{k}\left(\frac{x_{i}}{\left(x_{i}^{2}+y_{i}^{2}\right)^{1 / 2}}\right) \cos (\mathrm{k} \theta)\right.\right. \\
& \left.\left.\quad+\frac{y_{i}}{\left(x_{i}^{2}+y_{i}^{2}\right)^{1 / 2}} U_{k-1}\left(\frac{x_{i}}{\left(x_{i}^{2}+y_{i}^{2}\right)^{1 / 2}}\right) \sin (\mathrm{k} \theta)\right)\right]
\end{aligned}
$$

From local hypertoroidal moments $h_{e z i}$ and Cartesian coordinates $\left(x_{i}, y_{i}, z_{i}\right)$ of all sites $i$, function (6) interpolates the sum over radii and heights of all $h_{e z i}$ corresponding to azimut $\theta$. In Eq. (6), $T_{k}$ and $U_{k}$ respectively represent the $k$ th order Chebyshev polynomia of first and second kind.

It is observed that the dominant contribution of hypertoroidal moment oscillations comes from a sinusoidal component that has eight nodes. Thus, one could assign as a first approximation the $\overline{8}$ point group of symmetry to the polarization field lines pattern.

Returning to the homogeneously hypertoroidized phase III, one should pay attention to the size of simulated nanotori: Provided that extremely small objects can be experimentally obtained, the information density of a memory nanodevice using these individual particles as ferroelectric bits would be enormous. Moreover, in addition to the advantage of small size, the absence of overall polarization should strongly reduce the phenomenon of electrostatic interaction between neighboring ferroelectric bits, and the inhomogeneous electric field produced by these dipolar arrangements may allow nondestructive readout through noncontact electric force microscopy. One should also mention that $\overline{8}$ and $\infty / m$ (phase II) symmetry groups have an inversion center, whereas $\infty$ symmetry group (phase III) does not. This means that the low temperature phase III may be gyrotropic. Thus, it may exhibit an optical activity that could possibly be tuned by modulating hypertoroidal moment and/or toroidal moment. Such a behavior has recently been simulated in the case of an array of aligned $\mathrm{BaTiO}_{3}$ nanowires embedded in a $\mathrm{SrTiO}_{3}$ matrix [25]: Well-separated nanotori embedded in a matrix could offer an alternative route for an experimental realization of such an optical modulator that would have the ability to change from levorotatory to dextrorotatory optical behavior.

Thus, we checked that hypertoroidal moment could effectively be switched by application of homogeneous electric fields. As a test case, we considered a torus with aspect ratio $a=0.90$ at a temperature of $40 \mathrm{~K}$ so as to be sure that, in the absence of external electric field, phase III is the stable initial state. We then cycled an electric field along the $z$ axis and recorded expression (5) of hypertoroidal moment. The hysteresis loop in Fig. 3 clearly demonstrates that there are two enantiomeric stable states in the absence of

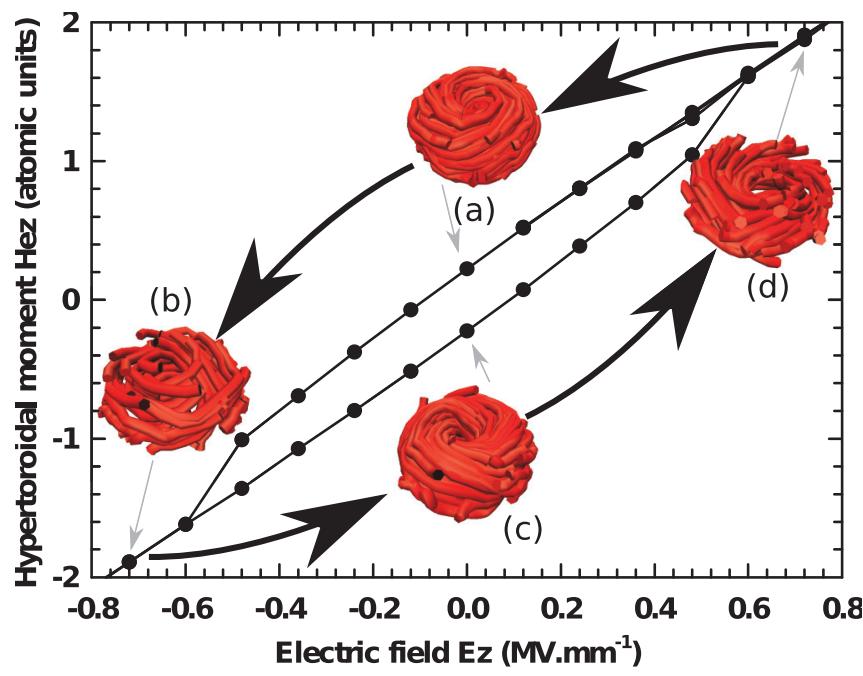

FIG. 3. (Color online) Hysteresis loop of axial hypertoroidal moment switched by homogeneous axial electric field for $a=0.90$ at $T=40 \mathrm{~K}$. Thick black arrows show the direction of the loop. Inset (a) represents the polarization field lines initial state at $40 \mathrm{~K}$ under zero field, which is the same as in Fig. 2(c). (b) is taken at $-7.2 \times$ $10^{8} \mathrm{~V} / \mathrm{m}$. (c) is under zero field and represents an enantiomer of (a) due to switching of hypertoroidal moment without affecting the toroidal moment. (d) is taken at $+7.2 \times 10^{8} \mathrm{~V} / \mathrm{m}$.

an applied electric field [Figs. 3(a) and 3(c)] and that a homogeneous electric field along the $z$ axis allows switching.

To summarize, we have (1) obtained $\mathrm{BaTiO}_{3}$ nanotori. Later on, we focused on numerical simulation of an isolated nanotorus of arbitrary aspect ratio and found two entirely new states in addition to the already known paraelectric (phase I) and homogeneously toroidized (phase II) states. Computing the axial hypertoroidal moment led to (2) observation of homogeneous hypertoroidization that coexists with axial homogeneous toroidization (phase III). An even more striking state (phase IV) was also observed (3) due to azimuthal oscillations of the local axial hypertoroidal moment. Lastly (4), control of the homogeneous hypertoroidal moment was demonstrated in phase III. This opens a new way to increase the density of information stored in memory nanodevices, and a new possibility to create ultrafast optically active components.

We thank Fabienne Karolak for her help during the synthesis of $\mathrm{BaTiO}_{3}$ nanotori, Françoise Garnier for the SEM picture, and Paul Haghi-Hastiani for the TEM one. G.T. and J.M.K. want to thank Dr. Bellaiche for a very useful discussion. G.T. thanks Ecole Normale Supérieure de Lyon for financial support. Three-dimensional visualizations were performed with VAPORGUI software [26].
[1] V. Dubovik and V. Tugushev, Phys. Rep. 187, 145 (1990).

[2] I. I. Naumov, L. Bellaiche, and H. Fu, Nature (London) 432, 737 (2004).

[3] J. F. Scott, Science 315, 954 (2007).

[4] S. Prosandeev and L. Bellaiche, J. Mater. Sci. 44, 5235 (2009).
[5] S. Prosandeev, I. Ponomareva, I. Kornev, and L. Bellaiche, Phys. Rev. Lett. 100, 047201 (2008).

[6] S. Prosandeev and L. Bellaiche, Phys. Rev. Lett. 101, 097203 (2008).

[7] L. Louis, P. Gemeiner, I. Ponomareva, L. Bellaiche, G. Geneste, W. Ma, N. Setter, and B. Dkhil, Nano Lett. 10, 1177 (2010). 
[8] L. Louis, I. Kornev, G. Geneste, B. Dkhil, and L. Bellaiche, J. Phys.: Condens. Matter 24, 402201 (2012).

[9] B.-K. Lai, I. Ponomareva, I. A. Kornev, L. Bellaiche, and G. J. Salamo, Phys. Rev. B 75, 085412 (2007).

[10] M. Anoufa, J. M. Kiat, I. Kornev, and C. Bogicevic, J. Appl. Phys. 113, 054104 (2013).

[11] S. Prosandeev and L. Bellaiche, Phys. Rev. B 75, 094102 (2007).

[12] Z. Deng, Y. Dai, W. Chen, X. Pei, and J. Liao, Nanoscale Res. Lett. 5, 1217 (2010).

[13] D. Byrne, A. Schilling, J. F. Scott, and J. M. Gregg, Nanotechnology 19, 165608 (2008).

[14] S. Zhang, L.-M. Peng, Q. Chen, G. H. Du, G. Dawson, and W. Z. Zhou, Phys. Rev. Lett. 91, 256103 (2003).

[15] I. Hasegawa, Y. Sakaniwa, and H. Shima, Surf. Sci. 601, 5232 (2007).

[16] W. Zhong, D. Vanderbilt, and K. M. Rabe, Phys. Rev. B 52, 6301 (1995).

[17] H. Fu and L. Bellaiche, Phys. Rev. Lett. 91, 257601 (2003).

[18] S. Prosandeev and L. Bellaiche, Phys. Rev. B 77, 060101 (2008).

[19] Origin-independent expression (7) is written as [18]

$$
\begin{aligned}
\mathbf{H}_{e}= & \frac{1}{4 N v} \sum_{i}\left[\mathbf{r}_{i} \times\left(\mathbf{r}_{i} \times \delta \mathbf{p}_{i}\right)+\mathbf{r}_{i} \times 2 v \mathbf{T}\right. \\
& \left.-\frac{1}{N} \sum_{j} \mathbf{r}_{j} \times\left(\mathbf{r}_{i} \times \delta \mathbf{p}_{j}\right)\right] .
\end{aligned}
$$

We can use Jacobi identity and change the order in which summations are performed, to recast the third term of Eq. (7) into Eq. (8):

$$
\begin{aligned}
& \frac{1}{4 N v} \sum_{i}\left[-\frac{1}{N} \sum_{j} \mathbf{r}_{j} \times\left(\mathbf{r}_{i} \times \delta \mathbf{p}_{j}\right)\right] \\
& =-\frac{1}{4 N v} \sum_{i}\left[\mathbf{r}_{i} \times 2 v \mathbf{T}\right] \\
& \quad+\frac{1}{4 N^{2} v} \sum_{j}\left[\mathbf{r}_{j} \times\left(\delta \mathbf{p}_{j} \times \sum_{i} \mathbf{r}_{i}\right)\right] .
\end{aligned}
$$

As we chose a special set of coordinates that imposes $\sum_{i} \mathbf{r}_{i}=\mathbf{0}$, the second and third terms of Eq. (7) cancel each other and yield Eq. (5).

[20] See Supplemental Material at http://link.aps.org/supplemental/ 10.1103/PhysRevB.89.220103 for a comparison between poloidal moment and axial hypertoroidization in Cartesian coordinates.

[21] A. A. Gorbatsevich and Y. V. Kopaev, Ferroelectrics 161, 321 (1994).

[22] J. F. Scott, Nat. Mater. 4, 13 (2005).

[23] X. H. Zhu, P. R. Evans, D. Byrne, A. Schilling, C. Douglas, R. J. Pollard, R. M. Bowman, J. M. Gregg, F. D. Morrison, and J. F. Scott, Appl. Phys. Lett. 89, 122913 (2006).

[24] M. Anoufa, J. M. Kiat, I. Kornev, and C. Bogicevic, Phys. Rev. B 88, 144106 (2013).

[25] S. Prosandeev, A. Malashevich, Z. Gui, L. Louis, R. Walter, I. Souza, and L. Bellaiche, Phys. Rev. B 87, 195111 (2013).

[26] See http://www.vapor.ucar.edu. 